\title{
Focus Review
}

\section{Spatial organization in bacterial chemotaxis}

\author{
Victor Sourjik ${ }^{1, *}$ and Judith P Armitage ${ }^{2, *}$ \\ ${ }^{1}$ Zentrum für Molekulare Biologie der Universität Heidelberg, \\ DKFZ-ZMBH Alliance, Im Neuenheimer Feld, Heidelberg, Germany \\ and ${ }^{2}$ Department of Biochemistry, Oxford Centre for Integrative \\ Systems Biology, University of Oxford, Oxford, UK
}

Spatial organization of signalling is not an exclusive property of eukaryotic cells. Despite the fact that bacterial signalling pathways are generally simpler than those in eukaryotes, there are several well-documented examples of higher-order intracellular signalling structures in bacteria. One of the most prominent and best-characterized structures is formed by proteins that control bacterial chemotaxis. Signals in chemotaxis are processed by ordered arrays, or clusters, of receptors and associated proteins, which amplify and integrate chemotactic stimuli in a highly cooperative manner. Receptor clusters further serve to scaffold protein interactions, enhancing the efficiency and specificity of the pathway reactions and preventing the formation of signalling gradients through the cell body. Moreover, clustering can also ensure spatial separation of multiple chemotaxis systems in one bacterium. Assembly of receptor clusters appears to be a stochastic process, but bacteria evolved mechanisms to ensure optimal cluster distribution along the cell body for partitioning to daughter cells at division.

The EMBO Journal (2010) 29, 2724-2733. doi:10.1038/

emboj.2010.178

Subject Categories: signal transduction

Keywords: chemotaxis; Escherichia coli; Rhodobacter

sphaeroides

\section{Bacterial two-component systems}

Most environmental sensing in bacteria is performed by the so-called two-component systems (see Bourret and Silversmith, 2010 and references therein for recent reviews on the two-component systems). A canonical bacterial twocomponent system is comparatively simple and consists of a sensory histidine kinase and a phosphorylatable response regulator (Figure 1A). The kinase typically spans the cyto-

\footnotetext{
*Corresponding authors. V Sourjik, Zentrum für Molekulare Biologie der Universität Heidelberg, Im Neuenheimer Feld 282, DKFZ-ZMBH Alliance, Heidelberg 69120, Germany. Tel.: + 49622154 6858; Fax: + 49622154 5894; E-mail: v.sourjik@zmbh.uni-heidelberg.de or JP Armitage, Department of Biochemistry, Oxford Centre for Integrative Systems Biology, University of Oxford, Oxford OX1 3QU, UK.

Tel.: + 441865 613293; Fax: + 441865 613338;

E-mail: judith.armitage@bioch.ox.ac.uk
}

Received: 11 June 2010; accepted: 7 July 2010 plasmic membrane and senses the external stimuli using its periplasmic domain (Cheung and Hendrickson, 2010). In most cases, the signal is transmitted to the cytoplasmic part of the kinase through a conformational change in the stable kinase dimer, which causes changes in the kinase ability to autophosphorylate at a conserved histidine residue. In contrast to typical receptor-associated tyrosine or serine/threonine kinases in eukaryotes, the autophosphorylation does not stabilize the histidine kinase in an active state that is able to further phosphorylate substrate proteins. Rather, the phosphoryl group is directly transferred from the kinase histidine residue to the aspartate residue of the response regulator, dephosphorylating the kinase at each cycle of response regulator activation. This ensures not only fast signal termination, but also prevents the type of signal amplification that is common in eukaryotes, whereby a single molecule of stably activated kinase can phosphorylate multiple downstream target proteins before being dephosphorylated by a specific phosphatase.

Two-component response regulators typically consist of two domains-a regulatory, or receiver, domain phosphorylated by the sensory kinase and an output domain that performs a physiological function inside the cell (Bourret, 2010). In most cases, the output domain is a transcription factor that is inhibited by the unphosphorylated regulatory domain. The phosphorylated state of the response regulator is often intrinsically unstable, decaying in a matter of minutes or even seconds. Dephosphorylation of the response regulator is frequently further enhanced by the respective sensory kinase, which may, therefore, have a dual kinase-phosphatase function depending on its activity state (Kenney, 2010).

Although most of the two-component kinases appear to be evenly distributed over the cell membrane (VS, unpublished), several cases of specific spatial organization have been established, for example for the pathway that controls the asymmetric division of Caulobacter crescentus (Thanbichler, 2009). This review will focus on the spatial organization of the pathways that control chemotaxis, which form the most prominent signalling structures known so far in bacteria. We will consider examples of Escherichia coli, in which chemotactic signalling has been most thoroughly studied, and Rhodobacter sphaeroides, which represents a higher level of spatial organization complexity.

\section{Strategy of bacterial chemotaxis}

Chemotaxis pathways mediate navigation of motile bacterial cells in chemical gradients, enabling bacteria to migrate towards higher concentrations of attractants, while avoiding repellents (Sourjik, 2004; Wadhams and Armitage, 2004; Vladimirov and Sourjik, 2009). The small size of a bacterial cell would make direct spatial detection of gradients inefficient (Berg and Purcell, 1977), and bacteria consequently 

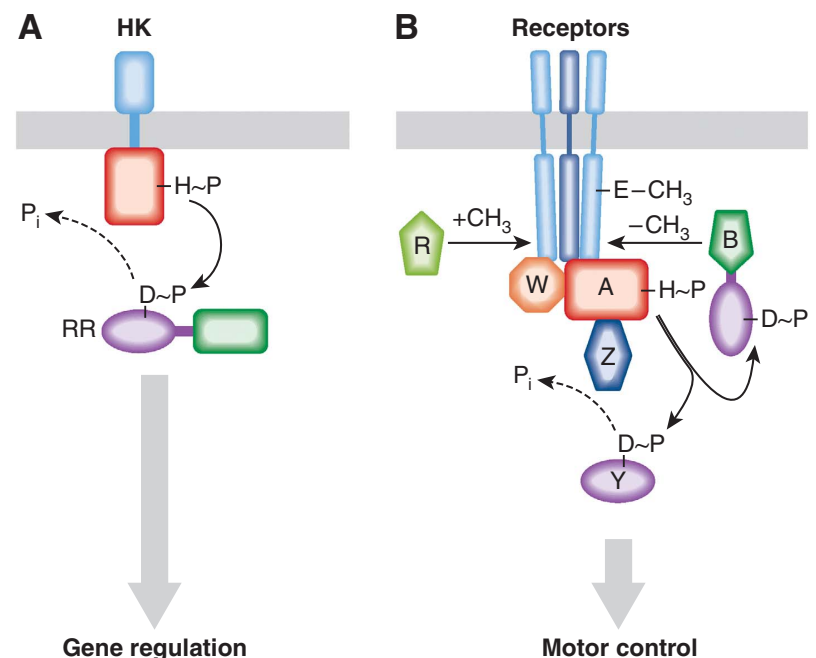

Figure 1 Two-component and chemotaxis signalling in bacteria. (A) Schematic representation of the canonical two-component system. Sensory histidine kinase $(H K)$ is composed of the input (blue) and the autokinase (red) domains; the kinase is typically a dimer. The response regulator $(R R)$ consists of the receiver (purple) and output (green) domains. The phosphate group is transferred from the histidine residue on the kinase to the asparate residue on the response regulator, activating the output domain, which typically regulates gene expression. The response regulator can be dephosphorylated by the phosphatase activity of the kinase. (B) Molecular composition of the chemotaxis pathway in E. coli. Receptors sense and transmit signals to regulate the activity of the cytoplasmic histidine kinase CheA. Receptors form trimers of dimers, where different types of receptors (light or dark blue) are mixed. CheA binding and regulation by receptors are aided by CheW. CheA transfers phosphate group to CheY, the single-domain response regulator controlling flagellar motor, and to CheB, composed of the regulatory receiver domain and the output methylesterase domain. Receptors are methylated on glutamate residues by the methyltransferase CheR. CheY is dephosphorylated by the phosphatase CheZ. Receptors, CheW, CheA and CheZ form a stable signalling core, to which CheR, CheB and CheY dynamically localize.

evolved a chemotaxis strategy that relies on temporal-rather than spatial-comparisons of chemoeffector concentrations (Berg and Brown, 1972; Macnab and Koshland, 1972). Bacteria moving in a gradient initially choose a swimming direction at random, but can then rapidly sense whether the chosen direction is favourable or not, and prolong the movement in a favourable swimming direction. Typically, the time for such temporal comparisons is limited to a few seconds, because on a longer time scale the cell would become reoriented by Brownian motion. The sensory pathway thus has to perform several tasks: (i) react to changes in chemoeffector concentration on a sub-second time scale; (ii) compare the level of stimulation at a given time point with that of $1-2 \mathrm{~s}$ earlier, which requires a short-term memory and (iii) gradually refresh the memory as the cell moves up or down the gradient. In E. coli, the general chemotaxis strategy is implemented by controlling the relative frequency of two swimming states: a smooth swimming 'run', which corresponds to the counterclockwise rotation of the flagellar motors and propels the cell forward, and re-orienting 'tumbles', which are produced by the clockwise (CW) motor rotation. In the adapted state with no gradients present, runs last $\sim 2 \mathrm{~s}$ and are interrupted by $\sim 0.1 \mathrm{~s}$ tumbles (Berg and Brown, 1972). As a result, cell performs a random walk that allows it to efficiently explore its environment. In the presence of a gradient, cells bias their random walk by suppressing tumbles, whereas swimming in a favourable direction, which results in an efficient net movement of a population up the gradient. Bacteria with single flagella achieve a similar random walk by either switching motor direction, resulting in a brief period of pulling rather than pushing the cell, or simply stopping flagellar rotation and letting Brownian motion reorient the cell for the next swimming period. The frequency regulation of reversals or stops produces the biased random walk.

This seemingly simple strategy has been greatly optimized through the course of evolution. Detailed quantitative analyses of $E$. coli chemotactic behaviour have shown that the response resulting in motor switching is extremely sensitive, with cells being able to detect minute changes in the level of stimulation (Segall et al, 1986), close to the physical limit set by the noise of ligand binding (Berg and Purcell, 1977). Cells can integrate multiple stimuli, enabling navigation in mixed gradients (Adler and Tso, 1974; Kalinin et al, 2010). Moreover, signalling by the pathway is robust against perturbations, such as variations in ambient ligands concentrations or in protein levels (Barkai and Leibler, 1997; Kollmann et al, 2005), which allows cells to maintain efficient chemotaxis in varying environments.

\section{Signalling in $E$. coli chemotaxis}

Given the complexity of its functions, it is not surprising that the chemosensory pathway in $E$. coli is much more sophisticated than a canonical two-component system (Figure 1B). Although signalling in chemotaxis similarly relies on a regulated phosphotransfer between a histidine kinase and a response regulator, the kinase CheA does not possess a sensory domain, but instead-together with the 'adaptor' protein CheW-associates with dedicated membrane-spanning receptors. E. coli has five types of such receptors, with four of them (Tar, Tsr, Tap and Trg) sensing not only a range of amino acids, sugars and dipeptides, but also $\mathrm{pH}$ and temperature, and the fifth (Aer) sensing redox potential. Changes in ligand binding to a periplasmic domain of receptors modulate conformation of their cytoplasmic parts (Hazelbauer et al, 2008; Hazelbauer and Lai, 2010) and consequently the autophosphorylation activity of receptorassociated CheA, whereby attractant binding decreases CheA activity and repellent binding enhances it. CheW is apparently required for this regulation, although its exact function remains unclear.

As in other two-component systems, the CheA phosphoryl group is subsequently transferred to the response regulator CheY, which can diffuse through the cytoplasm and transmit the signal, in this case to flagellar motors. CheY is an example of a single-domain response regulator, in which the output function-binding to the motor-is encoded in the receiver domain. Binding of phosphorylated CheY to the motors enhances the probability of CW rotation and thus causes the cell to tumble. When the cell swims up an attractant gradient, increased attractant stimulation results in CheA inactivation and suppression of tumbles, which promotes continued swimming in this direction. Rapid suppression of tumbles is further promoted by the sub-second dephosphorylation of CheY-P, which-in contrast to the canonical 
two-component sensors-is ensured by the dedicated phosphatase CheZ rather than by the phosphatase activity of the kinase itself.

Another unique feature of chemotaxis compared with the other two-component systems is its adaptability, which is mediated by methylation and demethylation of chemoreceptors on four specific glutamate residues by the methyltransferase CheR and the methylesterase CheB, respectively. Methylation tunes the ability of receptors to activate CheA, with higher modification increasing CheA activity and decreasing receptor sensitivity to attractants. Methylation thus resets the pathway activity upon an initial stimulation, counteracting the effects of ligand binding. Adaptation is achieved by at least two feedback mechanisms linking the activity of the ternary complex of receptors, kinase and CheW to the adaptation system. At a primary level, the feedback is provided by the substrate specificity of the adaptation enzymes, whereby CheR preferentially methylates inactive receptors and CheB preferentially demethylates active receptors (Barkai and Leibler, 1997; Alon et al, 1999; Amin and Hazelbauer, 2010). An additional negative feedback comes from a CheA-dependent phosphorylation of CheB, which possesses an inhibitory regulatory receiver domain. The kinetics of methylation and demethylation are relatively slow (Goy et al, 1977), because of the enzymatic properties of CheR and CheB and their low copy numbers. Depending on the initial stimulation strength, the adaptation time can take tens or hundreds of seconds, but for cells swimming in a typical gradient the methylation level lags behind changes in receptor activity by about a second (Brown and Berg, 1974; Berg and Tedesco, 1975). This lag confers the short-term memory to the chemotaxis system, which is required for temporal comparisons of attractant concentrations and is thus essential for chemotaxis.

\section{Spatial organization of $E$. coli chemotaxis pathway}

\section{Chemoreceptor clusters}

Although in vitro experiments suggest that the transmembrane signal transduction and kinase regulation in chemotaxis could be performed by small receptor-kinase complexes that consist of two to three receptor dimers, several CheWs and one CheA (Boldog et al, 2006; Amin and Hazelbauer, 2010), these complexes are organized in the cell into much larger macromolecular clusters that can contain thousands of receptors and associated chemotaxis proteins (Figure 2A). Receptor clusters are one of the largest structures in bacterial cells and can be easily observed at cell poles and along the cell body with immuno-electron, cryo-electron and fluorescence microscopy (Maddock and Shapiro, 1993; Sourjik and Berg, 2000; Zhang et al, 2007; Briegel et al, 2008). In these clusters, receptors are arranged in roughly hexagonal arrays (Figure $2 \mathrm{~B}$ and $\mathrm{C}$ ) that are presumably formed by trimers of receptor homodimers (Kim et al, 1999; Briegel et al, 2008, 2009; Khursigara et al, 2008). Clusters are further stabilized by the association of CheA and/or CheW (Maddock and Shapiro, 1993; Skidmore et al, 2000; Sourjik and Berg, 2000; Studdert and Parkinson, 2005; Kentner et al, 2006). Receptors of different ligand specificities appear to be uniformly mixed in the arrays, interacting with each other both directly and indirectly through CheA and CheW. Receptor
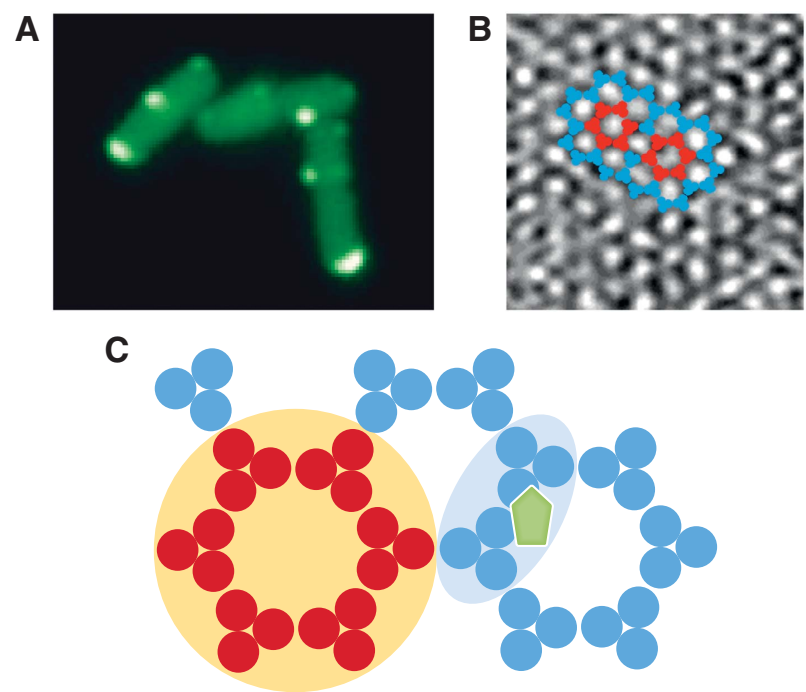

Figure 2 Receptor clusters in bacteria. (A) Fluorescence images of receptor clusters in $E$. coli cells. Images were obtained by expressing CheY fusion to yellow fluorescent protein (YFP) at the native location of the cheY gene on the chromosome. (B) Electron cryotomography images of receptor clusters in Vibrio cholerae, viewed from the 'top'. Cartoon illustrates fitting of trimers of dimers into the hexagonal lattice of a receptor array. Six trimers of dimers enclose one hexagon (red). Image is the courtesy of Ariane Briegel and Grant J Jensen, based on Briegel et al (2009). (C) Corresponding schematic representation of the receptor array, illustrating its functions in signal processing. According to the MWC model, receptors function in cooperatively switching signalling teams of 10-20 receptor dimers (yellow shading), whereby one team may correspond to a hexagon of the lattice (red). Adaptation enzymes tethered by binding to the pentapeptide sequence of receptors (CheR, green pentagon) can methylate or demethylate glutamates on $\sim 6$ receptors, creating an adaptation neighbourhood (light blue shading).

arrays are not perfectly regular structures: the hexagonal order appears to be frequently distorted (Khursigara et al, 2008) and the stoichiometry of the receptors to CheW and CheA can vary (Levit et al, 2002; Sourjik and Berg, 2004).

Interactions between receptors, CheW and CheA that are involved in the formation of the cluster core primarily occur at the cytoplasmic tip of the receptors (Miller et al, 2006; Park et al, 2006), although the exact arrangement of these proteins continues to be debated. All other chemotaxis proteins localize to the clusters by interaction with either receptors or CheA. CheR and CheB both bind to the NWETF pentapeptide sequence at the C-terminus of the major receptors, Tsr and Tar (Wu et al, 1996; Barnakov et al, 1999; Shiomi et al, 2002; Banno et al, 2004). CheZ interacts specifically with the $\mathrm{N}$-terminus of the short form of CheA, CheAs, that lacks the first 97 amino acids including the phosphorylation site (Sourjik and Berg, 2000; Cantwell et al, 2003; Cantwell and Manson, 2009; Hao et al, 2009; O’Connor et al, 2009). CheY associates with the cluster through binding to the P2 domain of CheA (Sourjik and Berg, 2000), which also binds CheB (Li et al, 1995).

\section{Cluster stability}

Fluorescence recovery after photobleaching experiments in vivo (Schulmeister et al, 2008) and biochemical measurements in vitro (Gegner et al, 1992; Erbse and Falke, 2009) showed that the sensory core of the cluster is stable on the 
time scale of chemotactic signalling. In vivo, receptors show no exchange between clusters even after $30 \mathrm{~min}$, whereas CheW and CheA exchange very slowly, with characteristic times of $12 \mathrm{~min}$, suggesting that signalling in chemotaxis is mediated by conformational changes in the cluster and not by its assembly or disassembly. Nevertheless, slow equilibration of the core components on the time scale of cell division could be beneficial, ensuring that the stoichiometry of sensory complexes is uniform throughout the cluster and between clusters, and that receptors of different specificities are well mixed. Yet another component of the stable cluster core is CheZ, with the exchange time of CheZ being $\sim 8 \mathrm{~min}$ (Schulmeister et al, 2008).

Other proteins associate with the cluster in a more dynamic way, in an apparent correspondence with their physiological function. The adaptation enzymes exchange between clusters on the time scale of $\sim 15 \mathrm{~s}$, which is much longer than the excitation time of the chemotaxis system $(\sim 0.1 \mathrm{~s})$, but is comparable with the time required for adaptation to saturating stimuli. Equilibration on this time scale may be essential to ensure a uniform distribution of adaptation enzymes-and thereby of receptor methylation states-in a cluster, as well as between multiple clusters in the same cell, particularly considering the low copy numbers (200-400) of CheR and CheB compared with $\sim 15000$ receptors (Li and Hazelbauer, 2004). On the other hand, a finite dwell time of the adaptation enzymes at the cluster appears to be important to allow fluctuations in the adapted level of kinase activity, optimizing bacterial search behaviour (Korobkova et al, 2004; Emonet and Cluzel, 2008; Matthaus et al, 2009). In contrast, CheY shows rapid cluster exchange kinetics on the time scale of several seconds, in concert with its function as messenger between the spatially localized sensory clusters and flagellar motors.

\section{Cluster assembly and positioning}

Recent experiments that determined the distribution of the size and number of receptor clusters and their dependence on the expression levels of chemotaxis proteins and on the cell length (Thiem and Sourjik, 2008; Greenfield et al, 2009) suggest that receptor clusters are formed by stochastic selfassembly, whereby individual receptors are inserted along the entire cell membrane (Shiomi et al, 2006) and subsequently either join existing clusters or nucleate new ones. Whereas the former process is primarily limited by receptor diffusion in the membrane and thus depends on the distance of the insertion site from the existing clusters, the latter is governed by the concentration of free receptors (Thiem and Sourjik, 2008). This interplay between concentration-dependent growth of old clusters and distance-dependent nucleation of new clusters results in the regular distribution of nucleated clusters along the cell body (Thiem et al, 2007; Thiem and Sourjik, 2008; Wang et al, 2008). Stochastic selfassembly can further account for the observed exponential distribution of receptor cluster sizes in photoactivated localization microscopy experiments (Greenfield et al, 2009).

Such mode of receptor cluster assembly couples cluster nucleation to cell body extension, such that additional clusters are nucleated away from the existing ones before a cell doubles its length and divides. This provides a simple and efficient mechanism to ensure an approximately uniform segregation of receptor complexes during cell division.
Receptor segregation appears to be additionally aided by the anchoring of larger lateral clusters to a putative periodic structure at future cell division sites (Thiem et al, 2007), possibly preventing cluster movement and accumulation on one side of the cell. As another consequence of anchoring at the division sites, clusters become positioned at a cell pole after a subsequent cell division. After division, clusters are apparently free to move, but remain restricted to the pole by some other mechanism, possibly the membrane curvature or lipid composition of the polar membrane. Membrane curvature is further likely to limit cluster size, destabilizing larger clusters because of the mismatch with the intrinsic curvature of receptor oligomers (Kim et al, 1999). Such mismatch may also explain the observed partial disorder in receptor arrays (Khursigara et al, 2008), which apparently consist of loosely connected small groups of well-arranged receptors.

The uniform cluster distribution along the cell body is not only important for cluster segregation, but it also benefits signalling. Uniform cluster distribution ensures that, even in longer cells, sites of CheY phosphorylation are not too remote from the laterally distributed flagellar motors, and the kinetics of chemotactic signalling is not limited by the diffusion of phosphorylated CheY (Sourjik and Berg, 2002a; Lipkow et al, 2004).

\section{Function of spatial organization in $E$. coli signal processing}

\section{Scaffolding and regulation}

What are the benefits of clustering for chemotaxis signalling? One obvious function-as in many other pathways-is to increase the efficiency of pathway reactions by raising local concentrations of all pathway components. Cluster assembly tends to position chemotaxis proteins favourably for their catalytic activities, and mutations that specifically affect protein localization can be compensated by overexpression of the affected proteins (Okumura et al, 1998; Jahreis et al, 2004). Localization seems to be particularly important for the function of the adaptation enzymes. Both CheR and CheB are able to modify several adjacent receptors while remaining tethered to one pentapeptide site, which is connected to the rest of the receptor by a flexible linker ( $\mathrm{Li}$ and Hazelbauer, 2006), creating the so-called adaptational assistance neighbourhoods (Figure 2C) with an estimated size of about six receptor dimers (Li and Hazelbauer, 2005). Such neighbourhoods appear to be important to ensure high precision of adaptation to a wide range of stimulus strengths (Endres and Wingreen, 2006), by providing each enzyme with a much larger number of available methylation sites -48 for a neighbourhood of six dimers instead of 8 for an individual dimerand thereby allowing a much more gradual adjustment of the overall receptor activity and preventing enzyme saturation.

Protein localization to the cluster can also have a subtler function. Although CheZ localization to the site of CheY phosphorylation seems counterintuitive, its importance seems to be in flattening the concentration gradient of phosphorylated CheY through the cell, so that flagellar motors at different distances from receptor clusters 'see' similar levels of phosphorylated CheY (Vaknin and Berg, 2004). Moreover, it is apparent that binding sites to the cluster for several proteins-CheA and CheW, CheR and CheB, and CheY and $\mathrm{CheB}$-overlap, and binding competition 
between individual proteins might have a regulatory function. For example, the competition between CheY and CheB (Li et al, 1995; Kentner and Sourjik, 2009) seems to strengthen the negative feedback from receptor activity to the methylation system, and may be important for robustness of the chemotaxis pathway (M Kollmann, personal communication).

\section{Signal amplification and integration by receptor arrays}

As mentioned above, the chemotactic response in $E$. coli is extremely sensitive, with cells responding to as little as $10 \mathrm{nM}$ steps in the concentration of aspartate (Mao et al, 2003), that is $<10$ molecules of aspartate in the 1.4 -fl volume of an $E$. coli cell. An increase in receptor occupancy by just $0.2 \%$ has been estimated to result in a $23 \%$ change in the bias of motor rotation (Segall et al, 1986), indicating signal amplification (or gain) by a factor of $\sim 100$. This amplification is achieved by highly cooperative behaviour of two macromolecular complexes that are involved in chemotaxis signalling: receptor arrays and flagellar motors. Interestingly, allosteric signal amplification by receptor arrays was initially proposed as a likely explanation of high sensitivity in chemotaxis based on purely theoretical considerations (Bray et al, 1998). Subsequent experimental studies confirmed the existence of cooperative interactions between receptors in vitro (Li and Weis, 2000; Lai et al, 2005) and in vivo (Gestwicki and Kiessling, 2002; Sourjik and Berg, 2004; Vaknin and Berg, 2008), and also showed that these interactions largely amplify chemotactic signals in vivo (Sourjik and Berg, 2002b, 2004).

These findings have provided a basis for the development of a number of detailed mathematical models describing cooperative behaviour of receptor arrays (Duke et al, 2001; Sourjik and Berg, 2004; Mello and Tu, 2005; Keymer et al, 2006), in which receptors in arrays switch cooperatively between active and inactive states, so that the activity of each individual receptor depends not only on the presence of a bound ligand molecule and on the level of receptor methylation, but also on the activities of neighbouring receptors. Most commonly used are the Monod-Wyman-Changeuxtype (MWC) models, which further assume that a cluster consists of many independent tightly coupled cooperative units, or signalling teams, of 10-20 receptor dimers (Figure 2C), with all receptors in one unit switching synchronously between inactive and active states (Sourjik and Berg, 2004; Mello and Tu, 2005; Keymer et al, 2006). Other, Isingtype, models describe the cluster as one lattice in which the activities of adjacent receptors are coupled with a finite strength of interaction, leading to an effective distance of the conformational spread (Duke and Bray, 1999; Mello and $\mathrm{Tu}, 2003)$. The number of effectively interacting receptors corresponds to the size of the cooperative unit in the case of the MWC model and to the extent of the conformational spread in the Ising model. It is likely that real receptor arrays may be better described by a hybrid model, in which groups of tightly coupled receptors are more loosely coupled to one another, consistent with the experimental analyses of the array structure (Khursigara et al, 2008).

In the MWC and Ising models, cooperative interactions between receptors in clusters can largely amplify changes in the kinase activity relative to ligand binding. If receptors have similar probability of being active or inactive, then additional inactivation of only a few receptors can stabilize the entire array in the inactive state. Moreover, as receptor homodimers with different ligand specificities appear to be randomly mixed in clusters (Studdert and Parkinson, 2004), cooperative interactions between them can ensure integration of different chemotactic signals. In the simplest case, the output of such a mixed array simply depends upon the total number of receptors occupied by ligand, allowing cells to regulate their chemoeffector preference by adjusting the numbers of receptors of particular type.

\section{Dynamics of signalling arrays}

Clustered organization of signalling proteins allows an additional level of regulation, through the composition and structure of the complex. Although the chemoreceptor arrays as a whole are stable on the time scale of signalling, local stimulation-induced rearrangements can occur, and the degree of cluster compactness indeed appears to be modulated by ligand stimulation and by sensory adaptation (Vaknin and Berg, 2007, 2008). Moreover, stimulation may also affect the stability and stoichiometry of the receptor clusters (Shrout et al, 2003). These effects seem to change the signalling properties of sensory complexes, with highly active receptors apparently working in larger signalling teams (Endres et al, 2008), which optimize the sensing properties of receptor clusters. Changes in cluster compactness may further affect the rates of phosphorylation and methylation in chemotaxis (Besschetnova et al, 2008), although the function of such regulation of chemotaxis signalling awaits further analysis.

\section{Signalling and spatial organization in $R$. sphaeroides chemotaxis}

Chemotaxis of $R$. sphaeroides represents the next level of complexity in the organization of the chemotaxis system. In contrast to E. coli, which primarily responds to the increase in attractant concentration, the major chemosensory response of $R$. sphaeroides is to a reduction in an attractant, such as an organic acid, oxygen or light. Another important difference is the requirement for transport of attractants for a normal chemosensory response, suggesting that metabolism at some level may be involved in the regulation of chemotaxis.

The sequenced genomes of $R$. sphaeroides strains all contain multiple putative chemotaxis pathway-encoding operons. The best-studied, $R$. sphaeroides WS8, has three operons, each encoding a complete pathway, with genes for putative chemoreceptors scattered around the two chromosomes. These chemotaxis pathways control a single stopping, rather than switching motor (Armitage and Macnab, 1987). Transcriptional studies suggest that one operon is not expressed under laboratory conditions (Poggio et al, 2007). However, all of the sensory proteins encoded by the other two operons are required for normal $R$. sphaeroides chemotaxis (Porter et al, 2002). These are three CheW, two CheA, two CheB, two CheR and three CheY proteins (Figure 3A). In each operon, while there are no genes for transmembrane receptors, there is a gene encoding a cytoplasmic receptor (transducer-like protein or Tlp). One operon, $\mathrm{CheOp}_{3}$, encodes a CheA homologue with the domains separated across two proteins, $\mathrm{CheA}_{3}$ and $\mathrm{CheA}_{4}$. CheA $\mathrm{A}_{4}$ has a receptor-binding domain and the kinase domain, whereas $\mathrm{CheA}_{3}$ has a receptor-binding domain and an Hpt domain, separated by a 
A
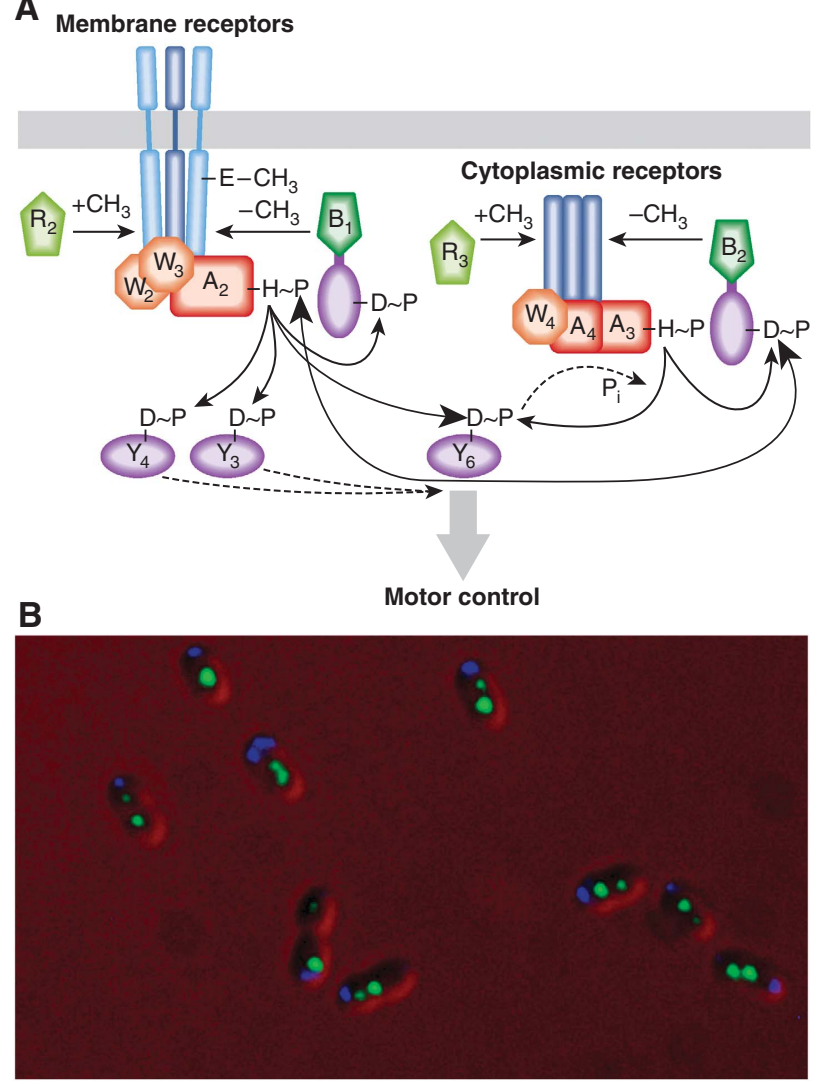

Figure 3 Spatial organization of the $R$. sphaeroides chemotaxis pathway. (A) The model of signalling in the $R$. sphaeroides chemotaxis. Attractants are thought to be sensed by the transmembrane chemoreceptors, which regulate activity of CheA2. Signals reflecting the metabolic state of the cell are believed to be sensed by the cytoplasmic receptors, regulating CheA4 and CheA3. CheY6 alone is capable of stopping the flagellar motor, but requires, in addition, either CheY3 or CheY4 to support chemotaxis. CheY6 is mainly phosphorylated by CheA3, but slow phosphotransfer from CheA2 has also been observed (illustrated using thin arrows). The phosphotransfer between CheA2 and CheB2 is reversible (indicated by the bi-sided arrow). (B) Polar and cytoplasmic clusters formed by the two chemosensory complexes. Fluorescence images show localization of the cyan fluorescent protein (CFP) fusion to CheW3 and YFP fusion to CheW4, expressed from endogenous genomic locations.

large unstructured domain, now know to be a specific phosphatase (Porter and Armitage, 2004). Early work using purified CheA and response regulator proteins showed that $\mathrm{CheA}_{2}$, encoded by CheOP2, could phosphotransfer to all $\mathrm{CheB}$ and $\mathrm{CheY}$ proteins in vitro (including those encoded by CheOp1 and not normally expressed), whereas $\mathrm{CheA}_{3} / \mathrm{CheA}_{4}$ could only phosphotransfer to the proteins encoded in the same operon, $\mathrm{CheB}_{2}$ and $\mathrm{CheY}_{6}$ (Porter and Armitage, 2002). Together, $\mathrm{CheA}_{3}$ and $\mathrm{CheA}_{4}$ form a functional histidine kinase and a phosphatase. The separation across two proteins may reflect a need to separate the kinase and phosphatase activity, while keeping the functions co-localized. Therefore, as in E. coli, the phosphatase is localized with the kinase, emphasizing the requirement to flatten the gradient of CheY-P (in this case $\mathrm{CheY}_{6}-\mathrm{P}$ ) in the cell.

Fluorescent fusions to $R$. sphaeroides chemotaxis proteins show that the transmembrane receptors show polar clustering similar to that of E. coli receptors, whereas the Tlps localize to either the middle of newly divided cells or the $\frac{1}{4}$ and $\frac{3}{4}$ positions of the dividing cells (Wadhams et al, 2002) (Figure 3B). When the other proteins were examined, $\mathrm{CheA}_{2}$, $\mathrm{CheW}_{2}, \mathrm{CheW}_{3}$ and $\mathrm{CheR}_{2}$, encoded by CheOp2, were all found to localize at the poles with the membrane receptors, whereas $\mathrm{CheA}_{3}$, $\mathrm{CheA}_{4}$, $\mathrm{CheW}_{4}$, $\mathrm{CheR}_{3}$, encoded by CheOp3, are all with the Tlps in the cytoplasmic cluster (Wadhams et al, 2003). Only one receptor in each cluster has a recognizable NWETF-like localization signal for CheR and, unlike E. coli, both CheBs appear delocalized, as do the three CheYs. Adaptation is clearly required, as it is in E. coli, but the mechanisms are unclear as the adaptation glutamates are difficult to identify on either membrane receptors or Tlps, although chemotaxis is reduced if putative glutamates are mutated to alanines. Deletion of either CheR or of $\mathrm{CheB}_{1}$ causes loss of chemotaxis, but deletion of $\mathrm{CheB}_{2}$ only reduces chemotaxis on swim plates, although individual tethered cells do fail to adapt (Martin et al, 2001). Unlike E. coli, deletion of the CheR or CheB proteins does not result in smooth swimming or stopped cells-free swimming cells still stop and start as wild type, but fail to respond. Only deletion of all three CheYs results in a smooth swimming phenotype. These data suggest that while the basic sensory system is similar to that of $E$. coli, there are major differences. Why are there two pathways and why and how are they physically separated within the cell?

There are several thousand receptors and CheW proteins within the clusters. In the cytoplasmic clusters, these must interact with the two CheA components, as these have different receptor-binding domains, the CheRs, and there must be access for the CheBs and CheYs. However, we know nothing of the organization of the proteins within the cytoplasmic cluster-whether they form an array of trimers of dimers, as seen for the polar clusters, and how these might coordinate-but we do know that the cluster is essential for normal chemotaxis and each daughter cell receives a cluster on cell division. If cells are treated with cephalexin to inhibit septation, long filamentous cells form, with the cytoplasmic clusters spaced at a cell length distance from each other, suggesting a highly controlled localization system (Wadhams et $a l, 2002)$. Any mutation that results in the cluster disassembling causes a loss of normal chemotaxis. It is, therefore, essential for accurate control of the flagellar motor behaviour that both pathways are functional, and each cell has both pathways.

As with $E$. coli, deletion of $\mathrm{CheW}_{2}, \mathrm{CheW}_{3}$ or $\mathrm{CheA}_{2}$ caused the transmembrane polar receptors to delocalize, and similarly deletion of $\mathrm{CheW}_{4}$ causes the cytoplasmic cluster to delocalize. Unlike E. coli, however, deletion of the Tlps, TlpT and $\mathrm{TlpC}$, also cause the cluster to dissociate, suggesting a function for the cytoplasmic receptors in cluster organization (Wadhams et al, 2005).

In the operon encoding the cytoplasmic cluster proteins, CheOp3, there is an unexpected gene encoding a protein PpfA, with homology to Soj from Bacillus subtilis and thus the ParA family of ATPases involved in plasmid segregation. The ParABS family control segregation of plasmids in a mechanism that is still unclear. ParA can form filaments when activated by ATP, and appears to oscillate over the chromosome. ParB anchors to a pars DNA sequence on the plasmid and is thought to trigger ParA depolymerization on contact, and this combined with non-specific chromosome binding by 
ParA helps segregate the plasmids as the cells segregate their chromosomes (Ringgaard et al, 2009). Intriguingly, when PpfA is deleted, no second cytoplasmic cluster is formed as the cell grows, although another forms in the daughter cell after cell division. In cephalexin-treated cells, there is just one, randomly positioned cluster in the long filaments (Thompson et al, 2006). Mutation of the Walker ATPase box in PpfA results in a similar phenotype to a deletion mutant. The $\mathrm{N}$ terminal domain of TlpT, encoded next to PpfA, has homology to ParB, and mutation of these sites also leads to the loss of cluster formation (JPA, unpublished). It, therefore, seems possible that a system related to one used to segregate plasmids may also ensure that each daughter cell has a cytoplasmic chemosensory pathway.

A search of sequenced genomes shows that $>50 \%$ genomes have more than one operon encoding a putative chemotaxis pathway. Of those with two or more operons, $>60 \%$ have one operon encoding a PpfA homology, usually next to a gene for a Tlp-like protein (Hamer et al, 2010). This suggests a common segregation system for cytoplasmic chemosensory proteins and that these large chemosensory protein clusters must be localized and segregated for correct function.

How related are the proteins in the polar and the cytoplasmic clusters? Overexpression of the CheW or the CheB proteins from $R$. sphaeroides in E. coli allowed at least partial complementation (Hamblin et al, 1997). However, unexpectedly, overexpression of, for example, $\mathrm{CheB}_{2}$ does not result in any complementation of a $\mathrm{CheB}_{1}$ deletion in $R$. sphaeroides (Ind et al, 2009). Indeed, none of the $R$. sphaeroides proteins appears able to complement the deletion of a homologue localized at a different position in the cell. Given the complexity of cluster architecture, it is possible that the homologous protein needs to form part of the cluster to complement any deletion. The P5 domains of the different CheA proteins determine their localization, whereas the P1 domains determine their phosphorylation target. The P5 receptor-binding domains were swapped between different CheAs, the constructs shown to be functional in vitro and to localize to the cluster dictated by the P5 domain. When the CheAs localized to the alternative cluster, chemotaxis was lost. When $\mathrm{CheA}_{2}$, known to phosphotransfer to all CheBs and CheYs, was localized to both clusters, there was still no chemotaxis (Scott et al, 2010). It seems that the individual pathways will only function with the correct proteins.

Interestingly, when $\mathrm{CheA}_{1}$, encoded by CheOp1 and not expressed under laboratory conditions, was expressed in either $\mathrm{CheA}_{2}$ or $\mathrm{CheA}_{3} / \mathrm{CheA}_{4}$ deletion backgrounds, it did not localize to either cluster, but stayed diffuse in the cell; indeed, overexpression of any polar or cytoplasmic cluster protein does not result in any protein localizing to the alternative cluster, suggesting a very specific architecture within the polar and cytoplasmic clusters, and much more specificity than that seen in E. coli.

This is borne out by recent co-crystal studies of $\mathrm{CheA}_{3} \mathrm{P} 1$ and its substrate $\mathrm{CheY}_{6}$. A single methionine present on the interaction interface of $\mathrm{CheY}_{6}$ and $\mathrm{CheB}_{2}$ determined the kinetics of phosphotransfer, and mutation to a serine, found in the other CheBs and CheYs phosphorylated by $\mathrm{CheA}_{2}$, but not $\mathrm{CheA}_{3}$, caused a loss in phosphotransfer, whereas the opposite mutation of the non-cognate CheYs leads to them becoming a substrate for $\mathrm{CheA}_{3}$ (Bell et al, 2010).
So why does $R$. sphaeroides need this complex and specific chemosensory organization? It is possible that the interplay between the polar and cytoplasmic pathways confers ability to make 'decisions' on responding to specific gradients dependent on the complex metabolic capabilities of a bacterial species normally living in nutrient poor conditions, but able to switch between aerobic, anaerobic and photosynthetic lifestyles. It is unclear what the environmental signals are that are sensed through the chemosensory cluster, as deletions of the cytoplasmic receptors result in cluster dissociation; however, one Tlp has a globin domain and mutations in TlpT lead to reductions in response to the major attractants, the organic acids. It, therefore, seems reasonable that the cytoplasmic receptors sense aspects of metabolic state, and these may regulate the response to signals coming through the transmembrane receptors at the cell poles. When the polar receptors are individually deleted, there is sequential reduction to most attractants, perhaps suggesting a complex interaction between the receptors in the polar lattice as indicated by the E. coli modelling and perhaps less ligand specificity.

As with E. coli, it appears not to be absolute copy number that matters, but maintaining the stoichiometry within the clusters allows sensing, and this may dictate the need for the complex architecture and organized localization system for the cytoplasmic cluster-overexpression of any individual component of the cytoplasmic cluster reduces chemotaxis, suggesting stoichiometry matters.

If this is the case and the cytoplasmic pathway regulates the strength of the signals through the polar cluster, there needs to be communication or integration of signals through the pathways. Experimental data show that $\mathrm{CheY}_{6}-\mathrm{P}$, which can be phosphorylated by either $\mathrm{CheA}_{2}$ or $\mathrm{CheA}_{3} / \mathrm{A}_{4}$, is the dominant motor-binding CheY, with $\mathrm{CheY}_{3}$ or $\mathrm{CheY}_{4}$ not able to stop the motor, but being required for chemotaxis, and, therefore, probably modulating the activity of $\mathrm{CheY}_{6}$ (Porter et al, 2006). There is no CheZ homologue in $R$. sphaeroides, but $\mathrm{CheY}_{6}-\mathrm{P}$, and only $\mathrm{CheY}_{6}-\mathrm{P}$, is dephosphorylated by the Hpt containing half-CheA, $\mathrm{CheA}_{3}$. Therefore, $\mathrm{CheA}_{4}$ is the kinase for only $\mathrm{CheY}_{6}$ and $\mathrm{CheB}_{2}$, but the level of $\mathrm{CheY}_{6}-\mathrm{P}$ is regulated by the activity of $\mathrm{CheA}_{3} . \mathrm{CheA}_{3}$ and $\mathrm{CheA}_{4}$ have different P5 domains and may therefore interact with different Tlps or have different responses to Tlp signalling. The concentration of $\mathrm{CheY}_{6}-\mathrm{P}$ is, therefore, dictated by the relative activity of $\mathrm{CheA}_{4}$ and $\mathrm{CheA}_{3}$, and this will directly control motor stopping frequency. But how is this modulated by the signals through the polar cluster? Computer modelling of the chemosensory pathway indicates that this is not at the level of the motor, where the CheYs could compete, but at the receptor cluster, with $\mathrm{CheB}_{2}-\mathrm{P}$, regulated by $\mathrm{CheA}_{3} / \mathrm{CheA}_{4}$, able to phosphotransfer to $\mathrm{CheA}_{2}$ and thus alter the signalling state of the polar cluster (Tindall et al, 2010).

\section{Evolutionary origins of the chemotaxis pathway organization}

It is interesting to speculate what has led to the observed structure and organization of the chemotaxis pathway. All of E. coli chemotaxis proteins, except CheZ, as well as their localization, are conserved among prokaryotes (Szurmant and Ordal, 2004; Wadhams and Armitage, 2004), suggesting that the observed pathway represents an evolutionary 
optimal solution. The important step in the emergence of the chemotaxis system from the canonical two-component pathway was separation of the sensor into multiple proteins. The primary evolutionary pressure for this process is likely to have come from the need to sense a large variety of ligands while feeding all the signals into the same signalling output, the task that is not easily accomplished by a single sensor. The resulting separation of receptors and kinase further simplified the evolution of new ligand specificities by receptor duplication and mutations without affecting the functionality of the existing system. On the other hand, it raised the problem of coupling multiple receptors to the same kinase, which was apparently solved by the appearance of the adaptor protein CheW-evolutionary related to the receptor-binding $\mathrm{C}$-terminal domain of $\mathrm{CheA}$ - and led to the emergence of receptor clustering. Subsequently, the allosteric interactions between receptors in clusters may have evolved,

\section{References}

Adler J, Tso WW (1974) Decision' making in bacteria: chemotactic response of Escherichia coli to conflicting stimuli. Science 184: $1292-1294$

Alon U, Surette MG, Barkai N, Leibler S (1999) Robustness in bacterial chemotaxis. Nature 397: 168-171

Amin DN, Hazelbauer GL (2010) The chemoreceptor dimer is the unit of conformational coupling and transmembrane signaling. J Bacteriol 192: 1193-1200

Armitage JP, Macnab RM (1987) Unidirectional, intermittent rotation of the flagellum of Rhodobacter sphaeroides. J Bacteriol 169: 514-518

Banno S, Shiomi D, Homma M, Kawagishi I (2004) Targeting of the chemotaxis methylesterase/deamidase $\mathrm{CheB}$ to the polar receptor-kinase cluster in an Escherichia coli cell. Mol Microbiol 53: 1051-1063

Barkai N, Leibler S (1997) Robustness in simple biochemical networks. Nature 387: 913-917

Barnakov AN, Barnakova LA, Hazelbauer GL (1999) Efficient adaptational demethylation of chemoreceptors requires the same enzyme-docking site as efficient methylation. Proc Natl Acad Sci USA 96: 10667-10672

Bell CH, Porter SL, Strawson A, Stuart DI, Armitage JP (2010) Using structural information to change the phosphotransfer specificity of a two-component chemotaxis signalling complex. PLoS Biol 8: e1000306

Berg HC, Brown DA (1972) Chemotaxis in Escherichia coli analysed by three-dimensional tracking. Nature 239: 500-504

Berg HC, Purcell EM (1977) Physics of chemoreception. Biophys J 20: 193-219

Berg HC, Tedesco PM (1975) Transient response to chemotactic stimuli in Escherichia coli. Proc Natl Acad Sci USA 72: 3235-3239

Besschetnova TY, Montefusco DJ, Asinas AE, Shrout AL, Antommattei FM, Weis RM (2008) Receptor density balances signal stimulation and attenuation in membrane-assembled complexes of bacterial chemotaxis signaling proteins. Proc Natl Acad Sci USA 105: 12289-12294

Boldog T, Grimme S, Li M, Sligar SG, Hazelbauer GL (2006) Nanodiscs separate chemoreceptor oligomeric states and reveal their signaling properties. Proc Natl Acad Sci USA 103: 11509-11514

Bourret RB (2010) Receiver domain structure and function in response regulator proteins. Curr Opin Microbiol 13: 142-149

Bourret RB, Silversmith RE (2010) Two-component signal transduction. Curr Opin Microbiol 13: 113-115

Bray D, Levin MD, Morton-Firth CJ (1998) Receptor clustering as a cellular mechanism to control sensitivity. Nature 393: $85-88$

Briegel A, Ding HJ, Li Z, Werner J, Gitai Z, Dias DP, Jensen RB, Jensen GJ (2008) Location and architecture of the Caulobacter crescentus chemoreceptor array. Mol Microbiol 69: $30-41$ enabling an exquisite sensitivity of the chemotaxis system, and further scaffolding functions have been added, depending on the specific chemotaxis system. In $R$. sphaeroides and other bacteria that evolved multiple sets of chemotaxis genes, cluster-based scaffolding has taken an additional function to spatially separate different chemotaxis pathways, in much the same way as scaffolding functions in eukaryotes.

\section{Acknowledgements}

Research in the VS lab has been supported by DFG and NIH and research in the JPA lab has been supported by BBSRC.

\section{Conflict of interest}

The authors declare that they have no conflict of interest.

Briegel A, Ortega DR, Tocheva EI, Wuichet K, Li Z, Chen S, Muller A, Iancu CV, Murphy GE, Dobro MJ, Zhulin IB, Jensen GJ (2009) Universal architecture of bacterial chemoreceptor arrays. Proc Natl Acad Sci USA 106: 17181-17186

Brown DA, Berg HC (1974) Temporal stimulation of chemotaxis in Escherichia coli. Proc Natl Acad Sci USA 71: 1388-1392

Cantwell BJ, Draheim RR, Weart RB, Nguyen C, Stewart RC, Manson MD (2003) CheZ phosphatase localizes to chemoreceptor patches via CheA-short. J Bacteriol 185: 2354-2361

Cantwell BJ, Manson MD (2009) Protein domains and residues involved in the CheZ/CheAS interaction. $J$ Bacteriol 191: 5838-5841

Cheung J, Hendrickson WA (2010) Sensor domains of two-component regulatory systems. Curr Opin Microbiol 13: 116-123

Duke TA, Le Novere N, Bray D (2001) Conformational spread in a ring of proteins: a stochastic approach to allostery. J Mol Biol 308: $541-553$

Duke TAJ, Bray D (1999) Heightened sensitivity of a lattice of membrane receptors. Proc Natl Acad Sci USA 96: 10104-10108

Emonet T, Cluzel P (2008) Relationship between cellular response and behavioral variability in bacterial chemotaxis. Proc Natl Acad Sci USA 105: 3304-3309

Endres RG, Oleksiuk O, Hansen CH, Meir Y, Sourjik V, Wingreen NS (2008) Variable sizes of Escherichia coli chemoreceptor signaling teams. Mol Syst Biol 4: 211

Endres RG, Wingreen NS (2006) Precise adaptation in bacterial chemotaxis through 'assistance neighborhoods'. Proc Natl Acad Sci USA 103: 13040-13044

Erbse AH, Falke JJ (2009) The core signaling proteins of bacterial chemotaxis assemble to form an ultrastable complex. Biochemistry 48: 6975-6987

Gegner JA, Graham DR, Roth AF, Dahlquist FW (1992) Assembly of an MCP receptor, CheW, and kinase CheA complex in the bacterial chemotaxis signal transduction pathway. Cell 70: 975-982

Gestwicki JE, Kiessling LL (2002) Inter-receptor communication through arrays of bacterial chemoreceptors. Nature 415: 81-84

Goy MF, Springer MS, Adler J (1977) Sensory transduction in Escherichia coli: role of a protein methylation reaction in sensory adaptation. Proc Natl Acad Sci USA 74: 4964-4968

Greenfield D, McEvoy AL, Shroff H, Crooks GE, Wingreen NS, Betzig E, Liphardt J (2009) Self-organization of the Escherichia coli chemotaxis network imaged with super-resolution light microscopy. PLoS Biol 7: e1000137

Hamblin PA, Bourne NA, Armitage JP (1997) Characterization of the chemotaxis protein CheW from Rhodobacter sphaeroides and its effect on the behaviour of Escherichia coli. Mol Microbiol 24: $41-51$

Hamer R, Chen PY, Armitage JP, Reinert G, Deane CM (2010) Deciphering chemotaxis pathways using cross species comparisons. BMC Syst Biol 4: 3 
Hao S, Hamel D, Zhou H, Dahlquist FW (2009) Structural basis for the localization of the chemotaxis phosphatase CheZ by CheAS. $J$ Bacteriol 191: 5842-5844

Hazelbauer GL, Falke JJ, Parkinson JS (2008) Bacterial chemoreceptors: high-performance signaling in networked arrays. Trends Biochem Sci 33: 9-19

Hazelbauer GL, Lai WC (2010) Bacterial chemoreceptors: providing enhanced features to two-component signaling. Curr Opin Microbiol 13: 124-132

Ind AC, Porter SL, Brown MT, Byles ED, de Beyer JA, Godfrey SA, Armitage JP (2009) Inducible-expression plasmid for Rhodobacter sphaeroides and Paracoccus denitrificans. Appl Environ Microbiol 75: 6613-6615

Jahreis K, Morrison TB, Garzon A, Parkinson JS (2004) Chemotactic signaling by an Escherichia coli CheA mutant that lacks the binding domain for phosphoacceptor partners. J Bacteriol 186: 2664-2672

Kalinin Y, Neumann S, Sourjik V, Wu M (2010) Responses of Escherichia coli bacteria to two opposing chemoattractant gradients depend on the chemoreceptor ratio. J Bacteriol 192: 1796-1800

Kenney LJ (2010) How important is the phosphatase activity of sensor kinases? Curr Opin Microbiol 13: 168-176

Kentner D, Sourjik V (2009) Dynamic map of protein interactions in the Escherichia coli chemotaxis pathway. Mol Syst Biol 5: 238

Kentner D, Thiem S, Hildenbeutel M, Sourjik V (2006) Determinants of chemoreceptor cluster formation in Escherichia coli. Mol Microbiol 61: 407-417

Keymer JE, Endres RG, Skoge M, Meir Y, Wingreen NS (2006) Chemosensing in Escherichia coli: two regimes of two-state receptors. Proc Natl Acad Sci USA 103: 1786-1791

Khursigara CM, Wu X, Subramaniam S (2008) Chemoreceptors in Caulobacter crescentus: trimers of receptor dimers in a partially ordered hexagonally packed array. J Bacteriol 190: 6805-6810

Kim KK, Yokota H, Kim SH (1999) Four-helical-bundle structure of the cytoplasmic domain of a serine chemotaxis receptor. Nature 400: 787-792

Kollmann M, Løvdok L, Bartholome K, Timmer J, Sourjik V (2005) Design principles of a bacterial signalling network. Nature 438: 504-507

Korobkova E, Emonet T, Vilar JM, Shimizu TS, Cluzel P (2004) From molecular noise to behavioural variability in a single bacterium. Nature 428: 574-578

Lai RZ, Manson JM, Bormans AF, Draheim RR, Nguyen NT, Manson MD (2005) Cooperative signaling among bacterial chemoreceptors. Biochemistry 44: 14298-14307

Levit MN, Grebe TW, Stock JB (2002) Organization of the receptorkinase signaling array that regulates Escherichia coli chemotaxis. J Biol Chem 277: 36748-36754

Li G, Weis RM (2000) Covalent modification regulates ligand binding to receptor complexes in the chemosensory system of Escherichia coli. Cell 100: 357-365

Li J, Swanson RV, Simon MI, Weis RM (1995) The response regulators $\mathrm{CheB}$ and $\mathrm{CheY}$ exhibit competitive binding to the kinase CheA. Biochemistry 34: 14626-14636

Li M, Hazelbauer GL (2004) Cellular stoichiometry of the components of the chemotaxis signaling complex. J Bacteriol 186: $3687-3694$

Li M, Hazelbauer GL (2005) Adaptational assistance in clusters of bacterial chemoreceptors. Mol Microbiol 56: 1617-1626

Li M, Hazelbauer GL (2006) The carboxyl-terminal linker is important for chemoreceptor function. Mol Microbiol 60: 469-479

Lipkow K, Andrews SS, Bray D (2004) Simulated diffusion of phosphorylated CheY through the cytoplasm of $E$. coli. J Bacteriol 187: 45-53

Macnab RM, Koshland Jr DE. (1972) The gradient-sensing mechanism in bacterial chemotaxis. Proc Natl Acad Sci USA 69: 2509-2512

Maddock JR, Shapiro L (1993) Polar location of the chemoreceptor complex in the Escherichia coli cell. Science 259: 1717-1723

Mao H, Cremer PS, Manson MD (2003) A sensitive, versatile microfluidic assay for bacterial chemotaxis. Proc Natl Acad Sci USA 100: 5449-5454

Martin AC, Wadhams GH, Shah DS, Porter SL, Mantotta JC, Craig TJ, Verdult PH, Jones H, Armitage JP (2001) CheR- and CheB- dependent chemosensory adaptation system of Rhodobacter sphaeroides. J Bacteriol 183: 7135-7144

Matthaus F, Jagodic M, Dobnikar J (2009) E. coli superdiffusion and chemotaxis-search strategy, precision, and motility. Biophys J 97: 946-957

Mello BA, Tu Y (2003) Perfect and near-perfect adaptation in a model of bacterial chemotaxis. Biophys J 84: 2943-2956

Mello BA, Tu Y (2005) An allosteric model for heterogeneous receptor complexes: understanding bacterial chemotaxis responses to multiple stimuli. Proc Natl Acad Sci USA 102: 17354-17359

Miller AS, Kohout SC, Gilman KA, Falke JJ (2006) CheA kinase of bacterial chemotaxis: chemical mapping of four essential docking sites. Biochemistry 45: 8699-8711

O'Connor C, Matsumura P, Campos A (2009) The CheZ binding interface of CheAS is located in alpha-helix E. J Bacteriol 191: $5845-5848$

Okumura H, Nishiyama S, Sasaki A, Homma M, Kawagishi I (1998) Chemotactic adaptation is altered by changes in the carboxyterminal sequence conserved among the major methyl-accepting chemoreceptors. J Bacteriol 180: 1862-1868

Park SY, Borbat PP, Gonzalez-Bonet G, Bhatnagar J, Pollard AM, Freed JH, Bilwes AM, Crane BR (2006) Reconstruction of the chemotaxis receptor-kinase assembly. Nat Struct Mol Biol 13: 400-407

Poggio S, Abreu-Goodger C, Fabela S, Osorio A, Dreyfus G, Vinuesa P, Camarena L (2007) A complete set of flagellar genes acquired by horizontal transfer coexists with the endogenous flagellar system in Rhodobacter sphaeroides. J Bacteriol 189: 3208-3216

Porter SL, Armitage JP (2002) Phosphotransfer in Rhodobacter sphaeroides chemotaxis. J Mol Biol 324: 35-45

Porter SL, Armitage JP (2004) Chemotaxis in Rhodobacter sphaeroides requires an atypical histidine protein kinase. $J$ Biol Chem 279: $54573-54580$

Porter SL, Wadhams GH, Martin AC, Byles ED, Lancaster DE, Armitage JP (2006) The CheYs of Rhodobacter sphaeroides. J Biol Chem 281: 32694-32704

Porter SL, Warren AV, Martin AC, Armitage JP (2002) The third chemotaxis locus of Rhodobacter sphaeroides is essential for chemotaxis. Mol Microbiol 46: 1081-1094

Ringgaard S, van Zon J, Howard M, Gerdes K (2009) Movement and equipositioning of plasmids by ParA filament disassembly. Proc Natl Acad Sci USA 106: 19369-19374

Schulmeister S, Ruttorf M, Thiem S, Kentner D, Lebiedz D, Sourjik V (2008) Protein exchange dynamics at chemoreceptor clusters in Escherichia coli. Proc Natl Acad Sci USA 105: 6403-6408

Scott KA, Porter SL, Bagg EA, Hamer R, Hill JL, Wilkinson DA, Armitage JP (2010) Specificity of localization and phosphotransfer in the CheA proteins of Rhodobacter sphaeroides. Mol Microbiol 76: 318-330

Segall JE, Block SM, Berg HC (1986) Temporal comparisons in bacterial chemotaxis. Proc Natl Acad Sci USA 83: 9486-9493

Shiomi D, Yoshimoto M, Homma M, Kawagishi I (2006) Helical distribution of the bacterial chemoreceptor via colocalization with the Sec protein translocation machinery. Mol Microbiol 60: 894-906

Shiomi D, Zhulin IB, Homma M, Kawagishi I (2002) Dual recognition of the bacterial chemoreceptor by chemotaxis-specific domains of the CheR methyltransferase. J Biol Chem 277: 42325-42333

Shrout AL, Montefusco DJ, Weis RM (2003) Template-directed assembly of receptor signaling complexes. Biochemistry 42: $13379-13385$

Skidmore JM, Ellefson DD, McNamara BP, Couto MM, Wolfe AJ, Maddock JR (2000) Polar clustering of the chemoreceptor complex in Escherichia coli occurs in the absence of complete CheA function. J Bacteriol 182: 967-973

Sourjik V (2004) Receptor clustering and signal processing in E. coli chemotaxis. Trends Microbiol 12: 569-576

Sourjik V, Berg HC (2000) Localization of components of the chemotaxis machinery of Esherichia coli using fluorescent protein fusions. Mol Microbiol 37: 740-751

Sourjik V, Berg HC (2002a) Binding of the Escherichia coli response regulator CheY to its target measured in vivo by fluorescence resonance energy transfer. Proc Natl Acad Sci USA 99: $12669-12674$ 
Sourjik V, Berg HC (2002b) Receptor sensitivity in bacterial chemotaxis. Proc Natl Acad Sci USA 99: 123-127

Sourjik V, Berg HC (2004) Functional interactions between receptors in bacterial chemotaxis. Nature 428: 437-441

Studdert CA, Parkinson JS (2004) Crosslinking snapshots of bacterial chemoreceptor squads. Proc Natl Acad Sci USA 101: 2117-2122

Studdert CA, Parkinson JS (2005) Insights into the organization and dynamics of bacterial chemoreceptor clusters through in vivo crosslinking studies. Proc Natl Acad Sci USA 102: 15623-15628

Szurmant H, Ordal GW (2004) Diversity in chemotaxis mechanisms among the bacteria and archaea. Microbiol Mol Biol Rev 68: 301-319

Thanbichler M (2009) Spatial regulation in Caulobacter crescentus. Curr Opin Microbiol 12: 715-721

Thiem S, Kentner D, Sourjik V (2007) Positioning of chemosensory clusters in E. coli and its relation to cell division. EMBO $J$ 26: $1615-1623$

Thiem S, Sourjik V (2008) Stochastic assembly of chemoreceptor clusters in Escherichia coli. Mol Microbiol 68: 1228-1236

Thompson SR, Wadhams GH, Armitage JP (2006) The positioning of cytoplasmic protein clusters in bacteria. Proc Natl Acad Sci USA 103: 8209-8214

Tindall MJ, Porter SL, Maini PK, Armitage JP (2010) Regulation of phosphotransfer kinetics is critical control step in a complex chemosensory network. PLoS Comp Biol (in press)

Vaknin A, Berg HC (2004) Single-cell FRET imaging of phosphatase activity in the Escherichia coli chemotaxis system. Proc Natl Acad Sci USA 101: 17072-17077
Vaknin A, Berg HC (2007) Physical responses of bacterial chemoreceptors. J Mol Biol 366: 1416-1423

Vaknin A, Berg HC (2008) Direct evidence for coupling between bacterial chemoreceptors. J Mol Biol 382: 573-577

Vladimirov N, Sourjik V (2009) Chemotaxis: how bacteria use memory. Biol Chem 390: 1097-1104

Wadhams GH, Armitage JP (2004) Making sense of it all: bacterial chemotaxis. Nat Rev Mol Cell Biol 5: 1024-1037

Wadhams GH, Martin AC, Porter SL, Maddock JR, Mantotta JC, King HM, Armitage JP (2002) TlpC, a novel chemotaxis protein in Rhodobacter sphaeroides, localizes to a discrete region in the cytoplasm. Mol Microbiol 46: 1211-1221

Wadhams GH, Martin AC, Warren AV, Armitage JP (2005) Requirements for chemotaxis protein localization in Rhodobacter sphaeroides. Mol Microbiol 58: 895-902

Wadhams GH, Warren AV, Martin AC, Armitage JP (2003) Targeting of two signal transduction pathways to different regions of the bacterial cell. Mol Microbiol 50: 763-770

Wang H, Wingreen NS, Mukhopadhyay R (2008) Self-organized periodicity of protein clusters in growing bacteria. Phys Rev Lett 101: 218101

Wu J, Li J, Li G, Long DG, Weis RM (1996) The receptor binding site for the methyltransferase of bacterial chemotaxis is distinct from the sites of methylation. Biochemistry 35: 4984-4993

Zhang P, Khursigara CM, Hartnell LM, Subramaniam S (2007) Direct visualization of Escherichia coli chemotaxis receptor arrays using cryo-electron microscopy. Proc Natl Acad Sci USA 104: $3777-3781$ 